hep-ph/9510308

UMN-TH-1411/95

TPI-MINN-95/29

UCSBTH-95-31

October 1995

\title{
New Constraints on Superpartner Masses
}

\author{
Toby Falk, ${ }^{1}$ Keith A. Olive, ${ }^{1}$ Leszek Roszkowski, ${ }^{1}$ and Mark Srednicki ${ }^{2}$ \\ ${ }^{1}$ School of Physics and Astronomy, University of Minnesota, Minneapolis, MN 55455, USA \\ ${ }^{2}$ Department of Physics, University of California, Santa Barbara, CA 93106, USA
}

\begin{abstract}
We consider the Minimal Supersymmetric Standard Model (MSSM) without imposing relations on the superpartner masses that arise in grand unified theories. Given an arbitrary pattern of superpartner masses (consistent with experimental constraints), it may happen that the scalar potential is actually unstable, even though all scalar masses-squared are positive at the weak scale $M_{W}$. This is most likely to happen if the running mass-squared in a "flat" direction in field space becomes negative at some scale $Q_{0}$ which is well below the GUT scale. In this case, either this pattern of masses is ruled out, or there must be new physics (beyond the MSSM) at or below the scale $Q_{0}$.
\end{abstract}


The appearance of supersymmetry at the electroweak scale is widely regarded as a likely possibility. The main problem in analyzing supersymmetric extensions of the Standard Model is the huge number of new parameters. Even in the Minimal Supersymmetric Standard Model (MSSM), we must specify mass matrices for the superpartners; these masses softly break supersymmetry, and are arbitrary unless we make some assumptions about their ultimate origin. Of course, some of these new parameters are restricted by experimental constraints, in particular, by the absence of flavor-changing neutral currents. Still, it is always necessary to make some sort of simplifying assumptions in order to deal with the remaining complexity.

One of the best motivated assumptions is that of conventional grand unification, with the further assumption that all squark and slepton masses are equal at the grand unified (GUT) scale [1, 2]. However, there are a number of reasons to doubt these assumptions. First of all, even in a GUT, there is no reason for the squark and slepton masses of different generations to be equal. In addition, superstring models need not involve conventional grand unification, and can in fact give very different mass patterns [3]. The low energy content of the theory is often enlarged, with extra U(1) gauge bosons and extra scalars (moduli fields), and of course their superpartners. Also, it may be that supersymmetry is broken by extra strong interactions (technicolor) at relatively low energies, leading to still more complicated scenarios [4].

Our purpose here is to point out that certain mass patterns for the superpartners cannot arise unless there is new physics (beyond the MSSM) well below GUT scale. To see this, consider using the renormalization group equations to run the superpartner masses-squared up from the weak scale. It may happen that some squark or slepton mass-squared parameters become negative at some scale $Q_{0}$ which is well below the GUT scale, apparently leading to large and disastrous vacuum expectation values for the corresponding fields. If this is the case, clearly this region of parameter space is ruled out, or we made a mistake in trusting the renormalization group equations up to the scale $Q_{0}$. This would imply the existence of new physics at or below the scale $Q_{0}$. However, there is an important caveat: the computed VEVs are not trustworthy if they are much less than the renormalization scale $Q>Q_{0}$ which we choose to employ. This is because there are large logarithms in the loop corrections which cannot be absorbed into the running parameters. If, on the other hand, the VEVs turn out to be roughly equal to (or greater than) $Q$, then disastrous breaking of color and/or electric charge can be expected to occur.

Constraints on the parameter space (most notably on the soft supersymmetry breaking trilinear couplings $A$ ) arising from the existence of charge and/or color breaking minima have been previously considered [5]. As far as we know, however, previous authors have 
considered only directions in field space involving VEVs of Higgs fields, whereas the specific example we consider involves squark fields only.

Our analysis will use methods used previously to study vacuum stability in the MSSM (e.g., [6] and references therein). There are two cases which can be considered separately. In the first, all the VEVs appear in directions in field space in which the tree-level potential has nonvanishing quartic terms. (There may also be cubic terms, but these will not be qualitatively important, and we will neglect them for simplicity.) Schematically, we have

$$
V=\frac{1}{2} m^{2}(Q) \phi^{2}+\frac{1}{4} \lambda(Q) \phi^{4}
$$

The scalar masses, $m^{2}(Q)$, are affected by their gauge and Yukawa interactions. For a given set of masses at the weak scale, interactions with gauginos drive the scalar masses down as one moves to higher energy scales whereas their Yukawa interactions tend to drive them up [2]. The Yukawa interactions are important only for stops (and perhaps for sbottoms and staus if $\tan \beta$ is large). Depending on the particular pattern of sfermion masses at the weak scale, it may happen that the interactions with gauginos in fact drives $m^{2}(Q)$ negative, in which case the scalar potential has a minimum at $\phi=v(Q)=\left[-m^{2}(Q) / \lambda(Q)\right]^{1 / 2}$. We will assume that $m^{2}(Q)$ is negative when $Q$ is greater than some particular scale $Q_{0}$.

The presence of the minimum at $\phi=v(Q)$ can be trusted only if it is stable with respect to radiative corrections; in general, at the one-loop level these are of the form $\Delta V \sim$ $v(Q)^{4} \ln \left[v(Q)^{2} / Q^{2}\right][7]$. Hence, the minimum at $\phi=v(Q)$ is trustworthy if $v(Q) \simeq Q$, where the one-loop corrections can safely be assumed to be small. Thus, the best value of $Q$ to use is the one which yields $v(Q)=Q$. If $Q_{0}$ is large, then it can happen that this equation has no solution. This indicates that the true, renormalization-group invariant value of the VEV is zero. In general, however, we usually find that there is, in fact, a solution to $v(Q)=Q$, and this means that either (1) the pattern of superpartner masses which results in a nonzero VEV is ruled out, or (2) new physics must appear at or below the scale $Q$, new physics which somehow forces the VEV back to zero.

The analysis outlined above is somewhat complicated, since there are many directions in field space to check. Luckily, we find that the strongest constraints arise in the second case: directions in field space for which $\lambda(Q)=0$ for all $Q$. This case is the simplest to analyze, and the MSSM has, in fact, many such "flat" directions. Let us denote by $m_{\|}^{2}(Q)$ the mass-squared along one of these flat directions. Then if a negative $m_{\|}^{2}(Q)$ is found for $Q$ greater than some value $Q_{0}$, the potential is unbounded below for large field values in the flat direction. This unboundedness might still be cured, but only by nonrenormalizable terms in the scalar potential, such as $V_{\mathrm{NR}}(\phi) \sim M^{4-n} \phi^{n}$, where $n>4$, and $M$ is a new mass 
scale (e.g., the Planck mass or an intermediate scale) corresponding to new physics. In this case, the field will acquire a VEV

$$
v(Q) \sim M^{(n-4) /(n-2)}\left[-m_{\|}^{2}(Q)\right]^{1 /(n-2)}
$$

and for $M>Q_{0}$, there will usually be a solution of $v(Q)=Q$.

There are many flat directions which in principle must be checked. We concentrate on a particular example, the direction $\tilde{u}_{R}^{r}=\tilde{s}_{R}^{g}=\tilde{b}_{R}^{b} \equiv v(Q)$ [ $]$. We expect this direction to provide particularly strong bounds, as it involves only squarks (whose masses run faster than the sleptons due to their strong coupling to gluinos) and it does not involve the stop mass (whose running is slowed by Yukawa interactions). For this case we have $m_{\|}^{2}=m_{\tilde{u}_{R}}^{2}+m_{\tilde{s}_{R}}^{2}+$ $m_{\tilde{b}_{R}}^{2}$, whose renormalization group equation is

$$
Q \frac{\mathrm{d} m_{\|}^{2}}{\mathrm{~d} Q}=\frac{1}{8 \pi^{2}}\left[-16 g_{3}^{2} M_{3}^{2}-\frac{8}{3} g_{1}^{2} M_{1}^{2}+2 h_{b}^{2}\left(m_{\tilde{q}_{L}}^{2}+m_{\tilde{b}_{R}}^{2}+m_{H_{1}}^{2}+A_{b}^{2}\right)\right],
$$

where $g_{1}$ is the standard model $U(1)$ coupling, $M_{i}$ is a gaugino mass, $h_{b}$ is the bottom-quark Yukawa coupling, and $A_{b}$ is the bottom-quark trilinear mixing parameter; we have neglected terms involving first and second generation Yukawa couplings $\llbracket$. The full set of RGEs can be found, for example, in [2, 9, 10]. If $\tan \beta$ (the ratio of the two Higgs VEVs) is not too large, then $h_{b}$ is small, and the term proportional to $h_{b}^{2}$ has a nearly negligible effect. We see that, as $Q$ increases, $m_{\|}^{2}$ decreases, and may become negative.

Eq.(3) can be solved in closed form by making use of the one-loop relations $M_{i}(Q) \propto$ $g_{i}^{2}(Q)$ and $g_{i}^{2}\left(Q_{1}\right)=g_{i}^{2}\left(Q_{2}\right) /\left[1-b_{i} g_{i}^{2}\left(Q_{2}\right) \ln \left(Q_{1} / Q_{2}\right) /\left(8 \pi^{2}\right)\right]$ with $b_{3}=-3$ and $b_{1}=+11$. Assuming that the physical (propagator pole) squark masses entering $m_{\|}^{2}$ are less than the gluino mass $M_{3}$ and the bino mass $M_{1}$, then the solution of eq.(3) is

$$
\begin{aligned}
m_{\|}^{2}(Q)=m_{\|}^{2} & -\frac{2}{\pi^{2}} g_{3}^{2}\left(M_{3}\right) M_{3}^{2} \ln \left(Q / M_{3}\right)\left\{\frac{1+3 g_{3}^{2}\left(M_{3}\right) \ln \left(Q / M_{3}\right) /\left(16 \pi^{2}\right)}{\left[1+3 g_{3}^{2}\left(M_{3}\right) \ln \left(Q / M_{3}\right) /\left(8 \pi^{2}\right)\right]^{2}}\right\} \\
& -\frac{1}{3 \pi^{2}} g_{1}^{2}\left(M_{1}\right) M_{1}^{2} \ln \left(Q / M_{1}\right)\left\{\frac{1-11 g_{1}^{2}\left(M_{1}\right) \ln \left(Q / M_{1}\right) /\left(16 \pi^{2}\right)}{\left[1-11 g_{1}^{2}\left(M_{1}\right) \ln \left(Q / M_{1}\right) /\left(8 \pi^{2}\right)\right]^{2}}\right\}
\end{aligned}
$$

where all masses on the right-hand side are physical (propagator pole) masses. The only undetermined factors are $g_{3}^{2}\left(M_{3}\right)$ and $g_{1}^{2}\left(M_{1}\right)$, which depend on the full spectrum of superpartner masses (via threshold effects).

\footnotetext{
${ }^{1}$ Note that for this flat direction we have a cancellation of the U(1) $D$-terms which must be included for generic patterns of scalar masses. These contributions are absent in GUT models, where one assumes that the scalar masses are all equal at some scale [2, 10].
} 
We have solved the full set of RGEs numerically, and in fig. (1), we show contours of constant $v(Q)=Q$ in the $m_{\|} / \sqrt{3}-M_{3}$ plane; note that the $m_{\|} / \sqrt{3}$ is the root-mean-square average of the three relevant squark masses. We have taken $\alpha_{3}\left(M_{Z}\right)=0.12, A_{b, t}=0$, the higgsino mass parameter $\mu=1000 \mathrm{GeV}, \tan \beta=3$, and the pseudoscalar Higgs mass $m_{A}=400 \mathrm{GeV}$. We have actually run the RGE's for several sets of parameter choices, and we find negligible variation with respect to $A, \mu$, and $\tan \beta$, if $\tan \beta \lesssim 10$. Also, we have taken $M_{1}=M_{2}=M_{3}$ as an illustrative choice; our results are not at all sensitive to the value of $M_{2}$, and sensitive to the value of $M_{1}$ only if $M_{1} \gg M_{3}$. Furthermore, we have taken $V_{\mathrm{NR}}(\phi)=\frac{1}{6} M^{-2} \phi^{6}$ with $M=M_{\mathrm{Pl}}=1.2 \times 10^{19} \mathrm{GeV}$. The value of $v(Q)$ which labels the contours depends on this choice, roughly as $v(Q) \propto M^{1 / 2}$, so that lowering $M$ implies new physics at a lower scale. However, the location of the uppermost contour does not depend on $M$. Above this contour, there is no solution of $v(Q)=Q$, and so there is no unphysical minimum of the scalar potential; these values of $m_{\|}$and $M_{3}$, given by the approximate formula

$$
m_{\|} / \sqrt{3} \gtrsim 0.7 M_{3}
$$

for $M_{3}<2000 \mathrm{GeV}$, are allowed. Below this contour, there is a solution of $v(Q)=Q$, and so either these values of $m_{\|}$and $M_{3}$ are ruled out, or new physics must appear at or below the scale $Q$ (which depends on the choice of $V_{\mathrm{NR}}$ ). The most exciting possibility is the eventually experimental discovery of squarks and gluinos with masses which do not satisfy eq. (5), as this would predict new physics (beyond the MSSM) at or below the corresponding value of $Q$ shown in fig. (1). Note that based on our constraint, we do not expect to find squark masses with $m_{\|}$lower than our lowest contour labeled $10^{3} \mathrm{GeV}$, as we do not expect new physics below this scale. In addition, for $M<M_{\mathrm{Pl}}$ our constraints on $m_{\|}$are strengthened. Similar constraints can also be found for other combinations of squarks and sleptons along other flat directions.

Our constraint involves fairly large squark masses, and these can have cosmological consequences. We must ensure that the lightest supersymmetric particle (LSP) has an efficient annihilation channel, so that their relic mass density does not overclose the universe. If this particle is a gaugino, annihilation via squark and slepton exchange is the dominant mechanism, and so the squarks and sleptons cannot all be too heavy [11]. The large sfermion masses implied by our constraints lead to a relic density of gauginos $\Omega_{\widetilde{\chi}} h^{2} \gg 1$ unless either some combination of squark and slepton masses are light while still satisfying the bound on $m_{\|}$or there is significant mixing among the stops providing us with a relatively light stop. (This possibility requires of course that the LSP is heavier than the top quark [12].) Thus, the commonly made ansatz of taking equal squark and slepton masses at the weak 
scale is cosmologically excluded unless $m_{\tilde{\chi}}>m_{t}$ and we have a light stop. When the LSP is a higgsino, for which annihilation through intermediate sfermions is not as important, these cosmological restrictions do not apply. However, for a Higgsino LSP, $\Omega_{\tilde{\chi}} h^{2} \ll 1$, unless $m_{\tilde{\chi}} \gtrsim 1 \mathrm{GeV}$.

To conclude, we have identified a new constraint on supersymmetric models which do not include conventional grand unification. The sparticle masses at the weak scale must be such that the squark and slepton fields do not acquire large VEVs. Charge and/or color breaking minima can occur if a squark or slepton mass-squared becomes negative at some scale $Q_{0}$. Directions in field space for which the quartic term vanishes (which are in fact common in supersymmetric models) are particularly susceptible to the formation of large VEVs. In general, if the effective potential at scale $Q$ predicts a VEV of a squark or slepton field $v(Q)$ such that $v(Q)=Q$, then new physics must appear at or below this value of $Q$.

\section{Acknowledgments}

We would like to thank D. Bödecker, B. Campbell, S. Chaudhuri, H. Haber, A. Kovner, S.P. Martin, M. Voloshin, and H. Weigert for helpful discussions. This work was supported in part by DOE grant DE-FG02-94ER-40823 and NSF grant PHY-91-16964.

\section{References}

[1] E. Witten, Nucl. Phys. B188 (1981) 513; N. Sakai, Z.Phys. C11 (1981) 153; S. Dimopoulos and H. Georgi, Nucl. Phys. B193 (1981) 150; L.E. Ibanez and G.G. Ross, Phys. Lett. B110 (1982) 215; L.E. Ibanez, Phys. Lett. B118 (1982) 73; J. Ellis, D.V. Nanopoulos and K.Tamvakis, Phys. Lett. B121 (1983) 123; L. Alvarez-Gaumé, J. Polchinski and M.B. Wise, Nucl. Phys. B221 (1983) 495.

[2] K. Inoue, A. Kakuto, H. Komatsu and S. Takeshita, Prog. Th. Phys. 68 (1982) 927.

[3] L. Ibanez, J. Kim, H. Nilles, and F. Quevedo, Phys. Lett. 191B (1987) 282; B. Greene, K. Kirklin, P. Miron, and G. Ross, Nucl. Phys. B292 (1987) 606; I. Antoniadis, J. Ellis, J. Hagelin, and D.V. Nanopoulos, Phys. Lett. B231 (1989) 65; J. Casas, E. K. Katehou, and C. Munoz, Nucl. Phys. B317 (1989) 171; A. Font, L. Ibanez, F. Quevedo, and A. Sierra, Nucl. Phys. B331 (1990) 421; B. Greene, Phys. Rev. D40 (1989) 1645; R. Arnowitt and P. Nath, Phys. Rev. D42 (1990) 2948. A. Faraggi, Phys. Lett. 278 (1992) 131. 
[4] M. Dine and A.E. Nelson, Phys. Rev. D48 (1993) 1277; M. Dine, A.E. Nelson, and Y. Shirman, Phys. Rev. D D51 (1995) 1362; M. Dine, A.E. Nelson, Y. Nir, and Y. Shirman, SCIPP 95/32, hep-ph/9507358.

[5] C.Kounnas, A.B. Lahanas, D.V. Nanopoulos and M. Quirós, Nucl. Phys. B236 (1984) 438; J.M. Frère, D.R.T. Jones and S. Raby, Nucl. Phys. B222 (1983) 11; J.F. Gunion, H.E. Haber and M. Sher, Nucl. Phys. B306 (1988) 1; J.A. Casas, A. Lleyda and C. Muñoz, FTUAM95/11, hep-ph/9507294.

[6] J.R. Espinosa and M. Quiros, Phys. Lett. B353 (1995) 257; J.A. Casas, J.R. Espinosa, and M. Quiros, Phys. Lett. B342 (1995) 171.

[7] S. Coleman and E. Weinberg, Phys.Rev. D7 (1973) 1888; S. Weinberg, Phys Rev. D7 (1973) 2887.

[8] I. Affleck and M. Dine, Nucl. Phys. B249 (1985) 361.

[9] M. Drees and M. Nojiri, Phys. Rev. D47 (1993) 376; W. de Boer, R. Ehret, W. Oberschulte, and D.I. Kazakov, IEKP-KA/94-05, hep-ph/9405342.

[10] S.P. Martin and M.T. Vaughn, Phys. Rev. D50 (1994) 2282.

[11] K.A. Olive and M. Srednicki, Phys. Lett. B230 (1989) 78; Nucl. Phys. B355 (1991) 208; K. Griest, M. Kamionkowski, and M.S. Turner, Phys. Rev. D41 (1990) 3565; L. Roszkowski, Phys. Lett. B262 (1991) 59.

[12] T. Falk, R. Madden, K.A. Olive and M. Srednicki, Phys. Lett. B318 (1993) 354. 


\section{Figure Captions}

Fig. 1) Contours of constant $v(Q)=Q$ as a function of the gluino mass $M_{3}$ and the root-mean-square average squark mass $m_{\|}$. We take a non-renormalizable operator of the form $V_{N R}=\frac{1}{6} \phi^{6} / M_{\mathrm{Pl}}^{2}$ to stabilize the scalar potential at high scales. Successive contours represent an increase by a factor of 10 in $v(Q)$. Regions below a contour are forbidden unless new physics appears below that scale. There are no solutions to $v(Q)=Q$ for $v \gtrsim 10^{10} \mathrm{GeV}$. 


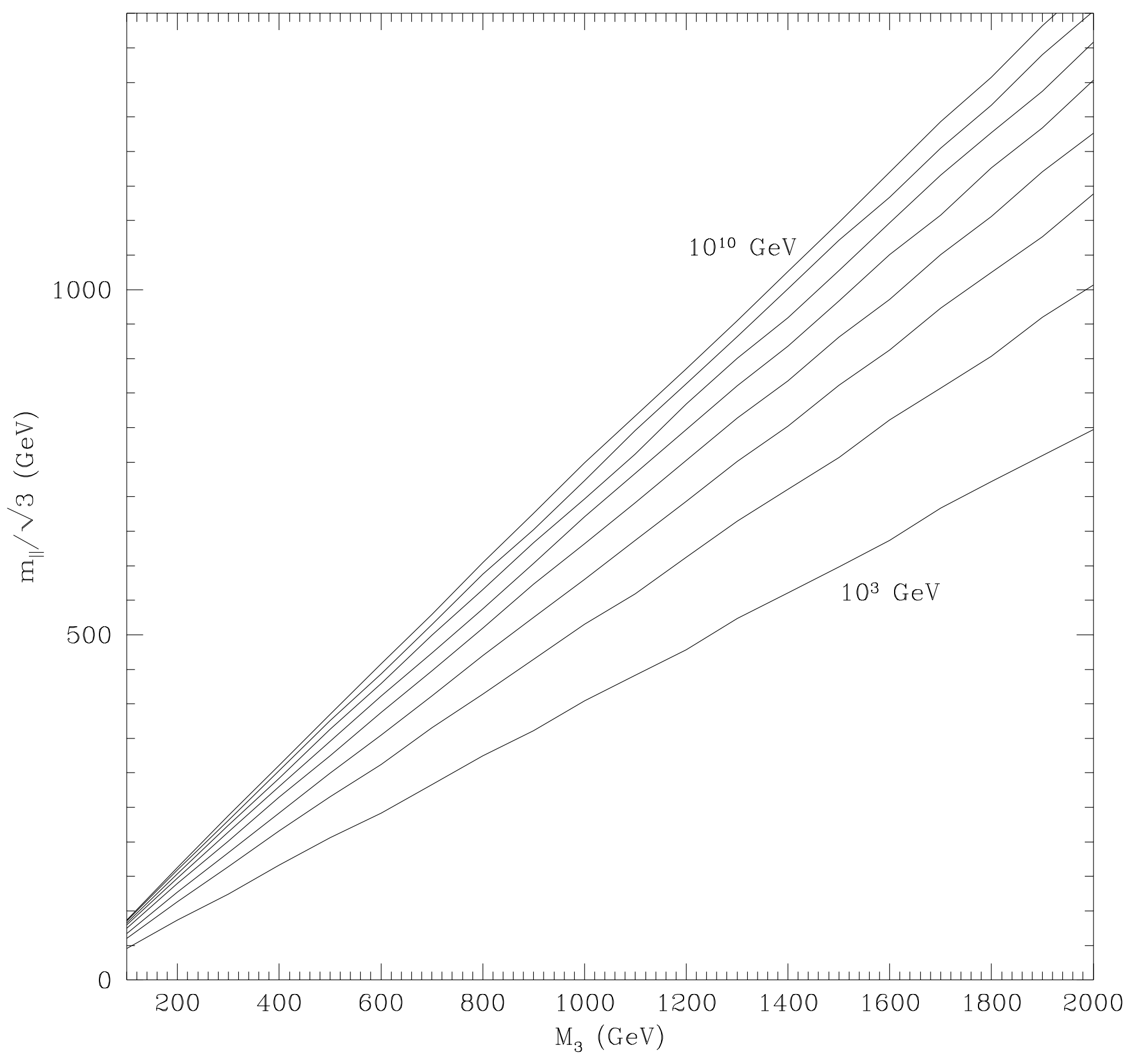

